INPLASY

PROTOCOL

To cite: Li et al. Baduanjin exercise for Lumbar Disc Herniation: A systematic review and meta-analysis. Inplasy protocol 2020110031. doi:

10.37766/inplasy2020.11.0031

Received: 07 November 2020

Published: 08 November 2020

Corresponding author:

Shusheng Guo

guossdongfang@163.com

Author Affiliation:

Dongfang College, Shandong

University of Finance and

Economics

Support: None.

Review Stage at time of this submission: Data analysis.

Conflicts of interest:

The authors declare that there is no conflict of interest regarding the publication of this paper.

\section{Baduanjin exercise for Lumbar Disc Herniation: A systematic review and meta-analysis}

Li, XK¹; Guo, SS2; Dong, H³; Zhou, LM4.

Review question / Objective: This meta-analysis of randomized controlled trials aims to evaluate the efficacy of Baduanjin for Lumbar Disc Herniation (LDH).

Condition being studied: Lumbar disc herniation (LDH) is a common disease in orthopedics, the incidence of which is about $2 \%$ to $5 \%$ in the general population, with the highest prevalence among people aged 30 to 50 , and the ratio of male to female is about 2:1. Due to its long course, easy repetition, difficult to cure, and accompanied by symptoms such as Lumbocrural pain, it seriously affects the quality of life of patients.

INPLASY registration number: This protocol was registered with the International Platform of Registered Systematic Review and Meta-Analysis Protocols (INPLASY) on 08 November 2020 and was last updated on 08 November 2020 (registration number INPLASY2020110031).

\section{INTRODUCTION}

Review question / Objective: This metaanalysis of randomized controlled trials aims to evaluate the efficacy of Baduanjin for Lumbar Disc Herniation (LDH).
Condition being studied: Lumbar disc herniation (LDH) is a common disease in orthopedics, the incidence of which is about $2 \%$ to $5 \%$ in the general population, with the highest prevalence among people aged 30 to 50 , and the ratio of male to female is about 2:1. Due to its long course, 
easy repetition, difficult to cure, and accompanied by symptoms such as Lumbocrural pain, it seriously affects the quality of life of patients.

\section{METHODS}

Participant or population: The patients were clinically diagnosed with lumbar disc herniation without surgical treatment.

Intervention: The exercise method selected during the intervention is the vertical Baduanjin.

Comparator: The intervention experiment group is a single Baduanjin or the control group is treated with Baduanjin, and the control group is the conventional conservative treatment of traditional Chinese and Western medicine, rehabilitation exercises, etc.

Study designs to be included: Randomized Controlled Trial (RCT), unlimited on gender, age, language, follow-up time.

Eligibility criteria: Studies with complete data and not republished.

Information sources: PubMed, the Cochrane Library, EMBASE, Web of Science, CNKI, and Wanfang Databases were searched to collect randomized controlled trials (RCTs) of Baduanjin in the treatment of LDH from the establishment of these databases to August 1, 2020.

Main outcome(s): Efficacy of Baduanjin for the treatment of lumbar disc herniation and mean changes in JOA and VAS scores at different intervention times.

Quality assessment / Risk of bias analysis: Two reviewers independently read the title and abstract according to the inclusion and exclusion criteria. Exclude obviously irrelevant documents, read the full text of the documents that may meet the requirements, determine whether they meet the standards and extract data, and check the outcome indicators of the included documents. The included studies were assessed for risk of bias in accordance with the requirements of the Cochrane Handbook for Systematic Reviews. If there is any ambiguity in the above operations, it can be solved by discussing or consulting a third researcher.

Strategy of data synthesis: Use RevMan $\mathbf{5 . 4}$ software provided by Cochrane Collaboration for statistical analysis. Relative risk (RR) was used to express the results of dichotomous data, and mean difference (MD) was used to express the results of continuous date, $95 \%$ confidence interval $(\mathrm{Cl})$ was calculated for each effect quantity.

Subgroup analysis: For the analysis of JOA and VAS scores, subgroup analyses were performed for different intervention times: within 1 month, 1 2 months, and more than 2 months.

Sensibility analysis: Sensitivity analysis by excluding literature one by one.

Country(ies) involved: China.

Keywords: Baduanjin; Lumbar Disc Herniation; Meta-analysis.

Contributions of each author:

Author 1 - Xiaokun Li.

Email: Ixkdfxy@163.com

Author 2 - Shusheng Guo.

Email: guossdongfang@163.com

Author 3 - Hong Dong.

Author 4 - Liming Zhou. 